\title{
Impact of the liquid level in the jet-film contact devices on the heat-and-mass transfer process
}

\author{
Oksana S. Dmitrieva ${ }^{1}$, Andrey V. Dmitriev ${ }^{1, *}$, Ilnur N. Madyshev ${ }^{2}$, and Leonid V. Kruglov ${ }^{1}$ \\ ${ }^{1}$ Kazan State Power Engineering University, 420066, Kazan, Krasnoselskaya str., 51, Russia \\ ${ }^{2}$ Kazan National Research Technological University, 420015, Kazan, Karl Marx str., 68, Russia
}

\begin{abstract}
For the purpose of increase in efficiency and performance of the heat-and-mass transfer processes, the design of jet-film contact device has been developed. The advantage of this device is a low hydraulic resistance, high mass-transfer efficiency with relatively low energy demands, as well as soiling resistance, efficient operation at low liquid loads. In this article the authors present the research results of heat-transfer from the flowing down liquid film to the gas flow inside of the proposed device. When calculating the Nusselt number, the authors used a correction coefficient, taking into account the impact of the liquid level in the device.
\end{abstract}

\section{Introduction}

Currently, the energy efficiency and conservation are a top five strategic courses of the priority technological development of Russia's economy. According to the International Finance Corporation and the World Bank, the energy consumption in Russia may be reduced in industrial infrastructure by more than twofold [1]. The strategic objective is the reduction of the energy intensity of the gross domestic product by not less than 2.3 times by 2030 [2]. In this regard, the relevant objective is to increase the energy conservation (saving) and energy efficiency of the industrial units of petrochemical plants. Different mass-transfer processes and apparatuses are applied in chemical, petrochemical and refining branches of industry. Basically, the efficiency of the behavior of such processes is determined by the perfection of the used contact devices, at that material and energy costs are strongly dependent on their design.

Nowadays, apparatuses with tray-type and packing contact devices are widely used in the industry, but have several disadvantages. In the tray-type column apparatuses it is necessary to provide separating zones, which significantly increase the vessel volume and, as a consequence, steel intensity. In the packing columns we can observe uneven distribution of liquid and gas phases in the cross section of the working area, which significantly reduces the efficiency of mass-transfer processes.

Heat-and-mass transfer equipment is the most promising for carrying out the processes of rectification under vacuum, absorption of gases at low pressures, as it has a low hydraulic resistance and high mass-transfer efficiency. Some research papers of the authors are devoted to study of the liquid flows in the mass-transfer apparatuses [3-6]. Many designs are inefficient from the point of view of achieving a high intensification of the processes at minimum energy demands. Therefore, it is necessary to develop contact devices, which can provide the most efficient and intense hydrodynamic modes with minimal hydraulic resistance.

\section{Description of the device and its operation}

The aim of this work is to develop effective apparatuses, based on the use of jet-film contact devices in relation to the processes of rectification, absorption, desorption and evaporation.

The authors have developed the jet-film contact device [7], consisting of parallel square drain cups with vertical walls (Fig. 1).

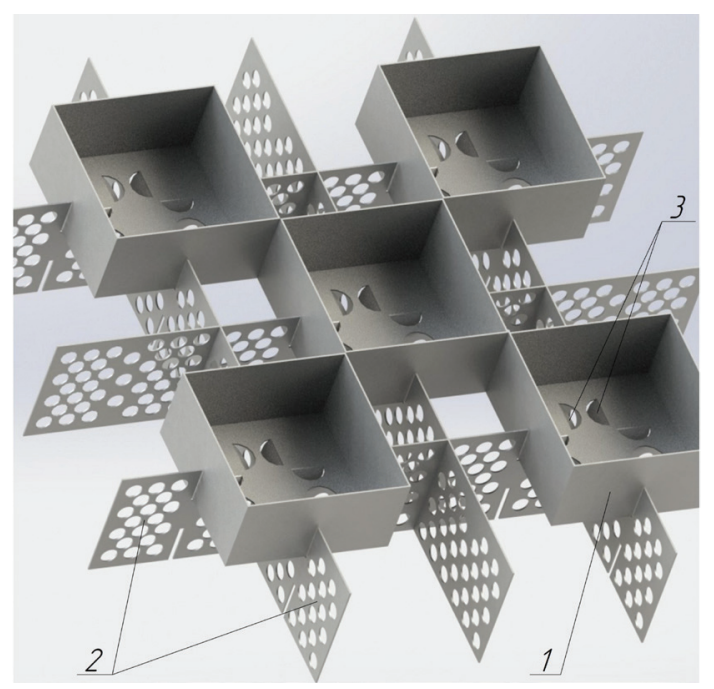

Fig. 1. One stage of the jet-film contact device: 1 - drain cup; 2 - perforated flow baffle plates; 3 - recurved leaves.

* Corresponding author: ieremiada@gmail.com 
Vertical walls, with slots for installation of appropriate drain cups, serve as supports. There are holes in the bottoms for intensification of the mass-transfer process at high values of the width of the drain cup $(b>$ $60 \mathrm{~mm}$ ). When liquid disperses through the holes, the jets disintegrate with drops formation. Thus, there are circular segments in the bottom of the device, which are curved to the bottom and required for the liquid distribution over the surface of the baffle plates. Each stage of the contact device is formed by a row of drain cups in a checkerboard order. Moreover, the downstream stage has an offset of drain cups, forming a vertical checkerboard order of them. The liquid is distributed with the formation of a stable film flow, when the liquid jets move over the surface of the baffle plates. Then, the formed film collides with the liquid surface, inside of the drain cups, breaks itself up and engages the gas volume inside. There is an impingement of drops, falling from the top plate, and a sharp decrease of the kinetic (motional) energy, which, in the ideal case, goes into the energy of formation of a bubble surface. For this reason, developed and constantly updated additional contact surface of phases is formed, which is determined by the presence of relatively small gas bubbles in the liquid layer and drops, departing from it.

In addition, the presence of holes in the baffle plates leads to the alignment of the profile of concentrations in the flowing down liquid film and its more uniform distribution over the surface, and to the decrease of the steel intensity of the proposed design.

The process of heat-and-mass transfer on the contact devices and in the apparatus as a whole is determined by the hydrodynamic environment. The study of hydrodynamic environment will help to understand the causes of qualitative changes of efficiency, to define the scope and specifics of operation, to identify further areas for the development engineering of the design of the device.

\section{Studies of heat-transfer from the flowing liquid film to the gas flow}

During the studies of heat-transfer from the flowing film of liquid to the gas flow, it was found that reduction of the liquid level in the drain cup causes the reduction of heat-transfer intensity up to $30 \%$. Consequently, for obtaining satisfactory results of calculation of the heattransfer from a flat baffle plate, we need to add a fudge (correction) factor that takes into account the impact of the liquid level in the drain cup.

The research was conducted on the example of heat exchange between air and a flat baffle plate. The air temperature was $20^{\circ} \mathrm{C}$, the temperature of the baffle plates was $40^{\circ} \mathrm{C}$. The width of the drain cup was $b=60$ $\mathrm{mm}$, and the height was $h_{1}=30 \mathrm{~mm}$. The liquid level in the drain cup $h_{2}$ was varied from 15 to $30 \mathrm{~mm}$. The research objective was to determine the average heattransfer coefficient from the flat baffle plate to the air flow. The velocity of the gases was varied in such a way that Reynolds number was varied in the range from 726 to $7.8 \cdot 10^{4}$.
For the analytic definition of average coefficients of momentum, heat and mass transfer, they widely use models of boundary layer (by Prandtl, Karman, Landau Levich, Taylor, Clauser, etc.). The quantitative correlation between friction and heat-transfer can be found using Chilton-Colburn analogy and its development [8]. Taking into account the damping of turbulent fluctuations in the viscous sublayer, the most promising is the model of diffusion boundary layer by Landau - Levich and other authors [9].

Criteria equation of the convection heat-transfer in the gas phase, in case of liquid flow over the flat baffle plate at turbulent flow regime, can be recorded as follows [10]:

$$
\mathrm{Nu}_{G}=0.037 \operatorname{Re}_{G}^{0.8} \operatorname{Pr}^{0.43}
$$

where $\mathrm{Nu}_{G}=\alpha b / \lambda-$ Nusselt number in the gas phase; $\alpha$ - heat-transfer coefficient, $\mathrm{W} /\left(\mathrm{m}^{2} \cdot \mathrm{K}\right) ; b$ - width of the contact element, $\mathrm{m} ; \boldsymbol{\lambda}$ - thermal conductivity coefficient, $\mathrm{W} /(\mathrm{m} \cdot \mathrm{K}) ; \operatorname{Pr}-$ Prandtl number.

Reynolds number in the gas phase was determined by the equation $\operatorname{Re}_{G}=b W_{G} / v_{G}$, where $W_{G}$ - actual gas velocity, $\mathrm{m} / \mathrm{s} ; \mathrm{v}_{G}$ - coefficient of kinematic gas viscosity, $\mathrm{m}^{2} / \mathrm{s}$.

\section{Research findings}

Research Findings The results of numerical studies are found overestimated (Fig. 2). The studies established that there are at least two modes of the heat-transfer, while the start of the second mode corresponds to the value of the Reynolds number $\operatorname{Re}_{G}=10^{4}$.

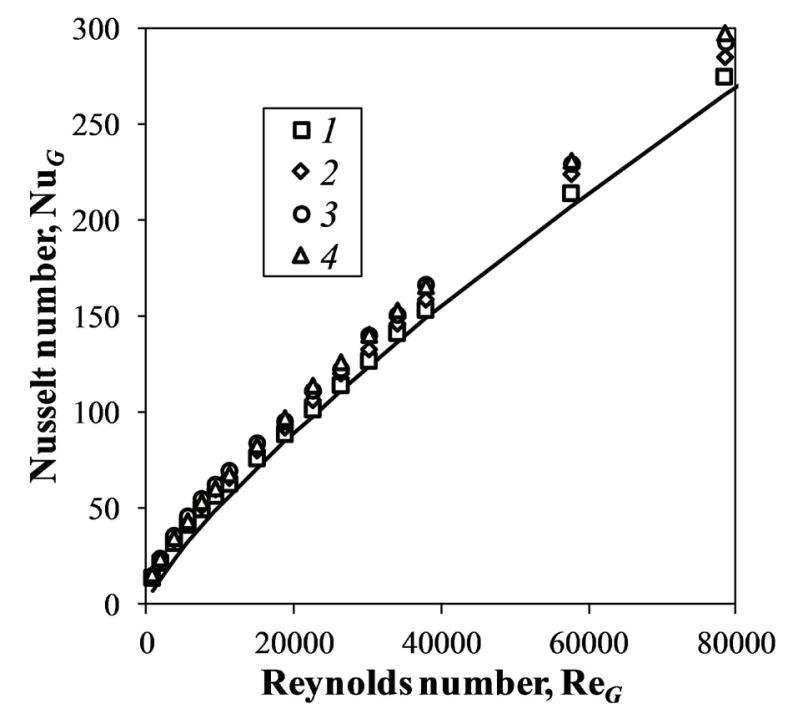

Fig. 2. The dependence of Nusselt number in the gas phase on Reynolds number at different liquid levels in the drain cup of the jet-film contact device $h_{2} / h_{1}=0.5(1) ; 0.666(2) ; 0.83(3)$; 1.0 (4); solid line - calculation according to the (Eq. 1), points - experiment, liquid - water.

To clarify the calculation of Nusselt number, taking into account the liquid level in the drain cup Nuh, we added the correction coefficient $\chi_{h}=\mathrm{Nu}_{h} / \mathrm{Nu}_{G}$, depending on the ratio of $h_{2} / h_{1}$, where $h_{1}$ - height of the 
vertical walls of the drain cups, $\mathrm{m} ; h_{2}$ - height of the liquid layer in the contact element, m. Using the calculated data in Fig. 3 as the base, we may record with high accuracy:

$$
\chi_{h}=1.16\left(h_{2} / h_{1}\right)^{0.16} \text {. }
$$

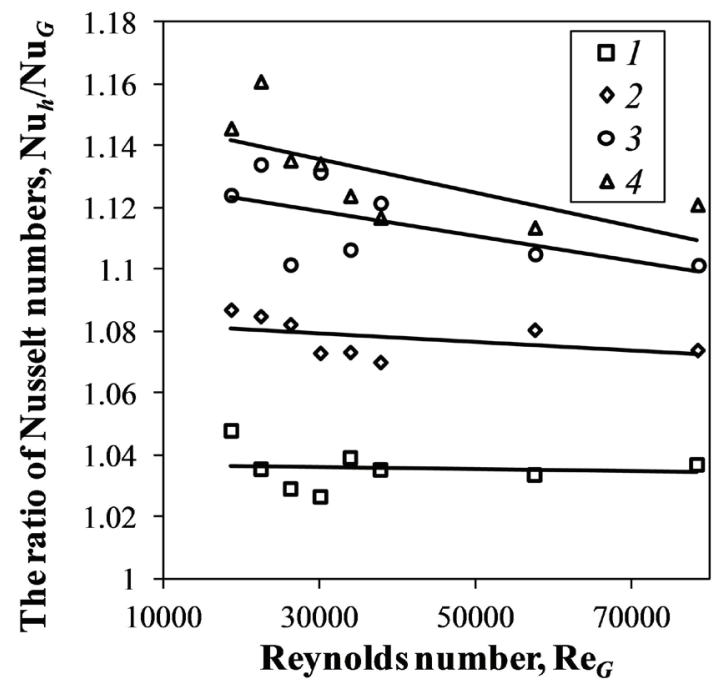

Fig. 3. The dependence of the ratio of Nusselt number, taking into account the liquid level in the drain cup, to Nusselt number in case of flowing around the flat plate, on Reynolds number at different ratio of $h_{2} / \mathrm{h}_{1}=0.5$ (1); 0.666 (2); 0.83(3); 1.0 (4); solid line - experience-based data fitting, points - experiment.

Then the average heat-transfer coefficient, when the flow is around the flat plate, can be determined from the modified criteria equation:

$$
N u_{h}=0.037 \chi_{h} \operatorname{Re}_{G}^{0.8} \operatorname{Pr}^{0.43} \text {, }
$$

where $\chi_{h}$ - coefficient, considering the impact of the liquid level in the drain cup.

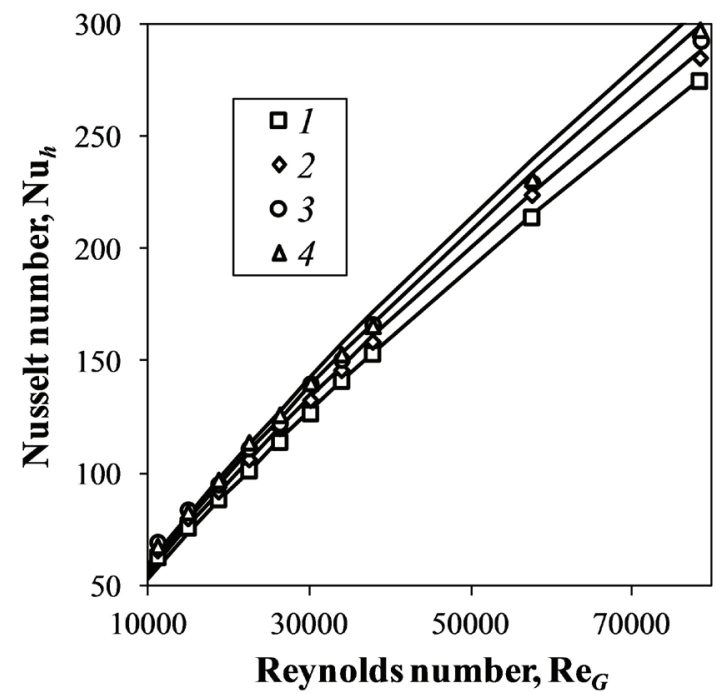

Fig. 4. The change of Nusselt number, obtained by the (Eq. 3), Reynolds number and different fill level of the drain cup $h_{2} / h_{1}$ $=0.5(1) ; 0.666(2) ; 0.83(3) ; 1.0(4)$; solid line - calculation according to the (Eq. 3), points - results of numerical simulation in Ansys Fluent.
Correlation of the data, obtained by the (Eq. 3) with the results of numerical simulation is shown in Fig. 4. The maximum values of heat transfer coefficients are observed when drain cups are completely filled. This is due to the lack of air flow expansion and as a result, a greater flow rate of air relative to the walls. The values of heat transfer coefficient increase by more than $30 \%$. The maximum thermal flow is observed at higher Reynolds numbers.

We have simulated the heat-transfer process from a flat baffle plate to the air. As shown in Fig. 5, relative error of calculation, according to the modified criteria equation, that takes into account the impact of the liquid level in the drain cup, does not exceed $\pm 9 \%$, in the range of the Reynolds numbers from $10^{4}$ up to $7.8 \cdot 10^{4}$. This is quite acceptable for engineering calculations.

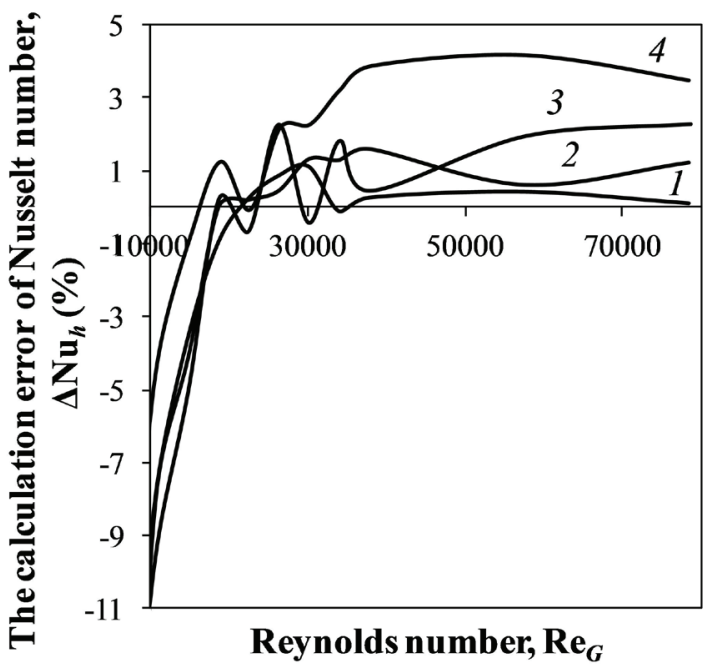

Fig. 5. The calculation error by the (Eq. 3) at different ratio $h_{2} / h_{1}=0.5(1) ; 0.666(2) ; 0.83(3) ; 1.0$ (4); the heat-transfer process from a flat baffle plate to the air.

To further verify the adequacy of calculations by the (Eq. 3), we have simulated the heat-transfer process from a flat baffle plate to the water. Underestimated values of Nusselt number, especially at high values of Reynolds number, were here observed. When using the fudge (correction) factor, the calculation accuracy was significantly increased (Fig. 6).

Calculations show that the proposed design can create the optimal conditions for a wide variety of heatmass transfer processes while providing a sufficiently high efficiency and relatively low energy costs for the organization processes. Developed delivery devices use energy of the liquid, flowing downwards, which reduces the flow resistance for the gas phase. In addition, by reducing the separation space, we can also reduce the size of units with the installed heat-mass transfer streambubble contact devices.

Thus, the proposed contact device should be designed so that the liquid level in the drain cups must be maximal. This is especially true for devices operating at sufficiently high gas velocities. 


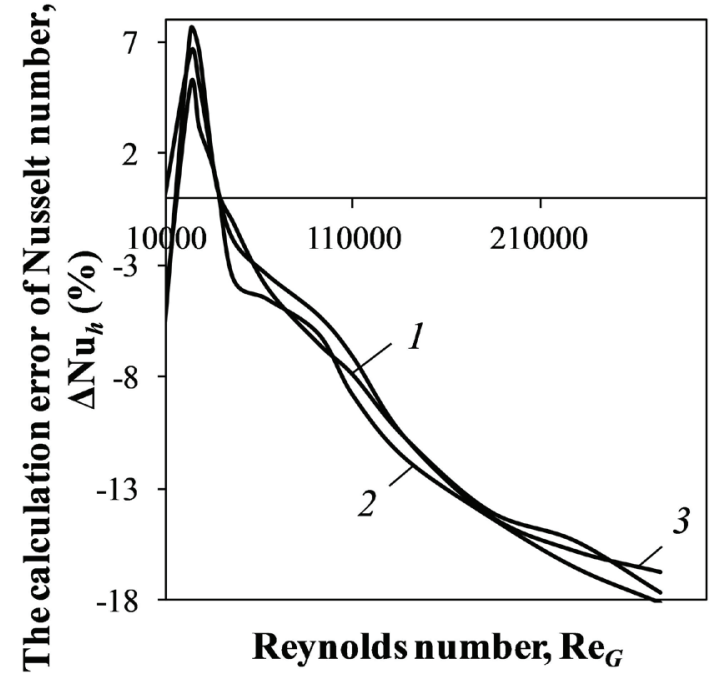

Fig. 6. The calculation error of Nusselt heat transfer number by the (Eq. 3) at different liquid levels in the drain cup of the jetfilm contact device $h_{2} / h_{1}=0.5$ (1); $0.666(2) ; 0.83(3)$; the heattransfer process from a flat baffle plate to the water.

\section{Conclusion}

On the basis of the conducted research we can draw the following conclusions:

1) organization of the original interaction between gas and liquid allows to intensify the mass-transfer processes both in liquid and in gas phases with a relatively simple design of the device;

2) contact of the phases is based on using the energy of the flowing down liquid stream, eliminating the necessity of redistribution of the liquid over the cross section of the apparatus;

3) modified criteria equation for the calculation of the average heat transfer coefficient from the flowing down liquid film to the gas flow, takes into account the impact of the liquid level in the drain cup;

4) jet-film contact devices will allow to create energyefficient apparatuses with a wide range of stable operation for rectification, absorption of gases.

The reported study was funded by RFBR, according to the research project No. 16-38-60081 mol_a_dk.

\section{References}

1. Energetic Efficiency in Russia. Burried Reserve. An account prepared by experts of world bank, International finance corporation and Center of effective use of energy, 2008. http://www.cenef.ru/file/FINAL_EE_report_rus.pdf.

2. Energetic Strategy of Russia for the Period to 2020, Approved by Russian Federation Government Order No. 1715-r on November 13, 2009.

3. G.Q. Wang, X.G. Yuan, and K. Yu, Review of Mass-Transfer Correlations for Packed Columns, Ind. \& Eng. Chem. Res., 44, 23, 8715 (2005).

4. A. Senol, U. Dramur, Performance test and design considerations of a column packed with a new ceramic packing, Chim. Acta turc, 23, 2, 145-155 (1995).

5. A.V. Dmitriev, O.S. Makusheva, K.V. Dmitrieva, and A.N. Nikolaev, Contact mass exchanger to increase output of active tower units, Chem. and Petrol. Eng., 47, 5-6, 319-323 (2011).

6. J. Maćkowiak, Model for the prediction of liquid phase mass transfer of random packed columns for gas-liquid systems, Chem. Eng. Res. and Des., 89, 8, 1308-1320 (2011).

7. A.V. Dmitriev, O.S. Dmitrieva, and I. N. Madyshev, Determination of the Mass-Transfer Coefficient in Liquid Phase in a Stream-Bubble Contact Device, Therm. Eng., 63, 9, 674-677 (2016).

8. A.P. Sinha, De Parameswar, Mass Transfer: Principles and Operations (PHI Learning Private Limited, New Delhi, 2012).

9. L.D. Landau, E.M. Lifshitz, Fluid Mechanics. Vol. 6 of Course of Theoretical Physics (ButterworthHeinemann, UK, 1987).

10. F.F. Tsvetkov, B.A. Grigorev, Teplomassoobmen [Heat and mass transfer] (MPEI Publ., Moscow, 2011). 